\title{
Coefficients in the CAVI Equation and the Comparison Between CAVI With and Without the Coefficients Using Clinical Data
}

\author{
Koji Takahashi ${ }^{1}$, Tomoyuki Yamamoto ${ }^{1}$, Shinichi Tsuda ${ }^{1}$, Fumio Okabe ${ }^{2}$, Tadashi Shimose ${ }^{2}$, Yoshinori Tsuji ${ }^{2}$, \\ Kenji Suzuki ${ }^{3}$, Kuniaki Otsuka ${ }^{4}$, Masanobu Takata ${ }^{5}$, Kazuhiro Shimizu ${ }^{6}$, Junji Uchino ${ }^{7}$ and Kohji Shirai ${ }^{7}$
}

${ }^{1}$ Fukuda Denshi Co., Ltd., Tokyo, Japan

${ }^{2}$ Osaka Hospital Anti-Tuberculosis Association, Osaka, Japan

${ }^{3}$ Japan Health Promotion Foundation, Tokyo, Japan

${ }^{4}$ Department of Internal Medicine, Tokyo Women's Medical University, Tokyo, Japan

${ }^{5}$ Toyama Teishin Hospital, Toyama, Japan

${ }^{6}$ Department of Internal Medicine, Toho University Sakura Medical Center, Sakura, Japan

${ }^{7}$ Mihama Hospital, Chiba, Japan

Aim: The Cardio-Ankle Vascular Index (CAVI) is a stiffness index of the arterial tree from the origin of the aorta to the ankle, independent of blood pressure at the time of measurement. The CAVI equation includes the coefficients "a" and "b" to adjust it to the value of Hasegawa's pulse wave velocity, which is compensated for at 80 $\mathrm{mmHg}$ of diastolic pressure. To verify this adjustment with the coefficients, the clinical significance of CAVI and CAVI without the coefficients (ha $\beta$ ) were compared in both an epidemiological study and an acute clinical study.

Methods: In the epidemiological study, the significances of CAVI and ha $\beta$ among people with or without coronary risks such as hypertension, dyslipidemia, hyperglycemia, and abnormal electrocardiography change, were compared. In the acute clinical study, nitroglycerin was administered to subjects in a control group and to coronary artery disease patients, observing CAVI and ha $\beta$ values over a 20 -min period.

Results: There was no discrepancy in terms of statistically significant differences between CAVI and ha $\beta$ among subjects with or without risk factors. Furthermore, there was also no discrepancy in terms of statistically significant differences between CAVI and ha $\beta$ during the changes of those values following nitroglycerin administration over a 20 -min period.

Conclusion: In both the epidemiologic and clinical studies, there was no discrepancy in terms of significant differences between CAVI and ha $\beta$. These results suggest that both are valid as indices of stiffness of the arterial tree from the origin of the aorta to the ankle.

Key words: Hasegawa's pulse wave velocity, Stiffness Parameter $\beta$, CAVI, ha $\beta$

\section{Introduction}

Arterial stiffness is a surrogate marker of arteriosclerosis and is established as a good predictor of cardiovascular events ${ }^{1-3)}$. Several methods have been designed to assess arterial stiffness, including pulse wave velocity (PWV), and plenty of valuable data have been reported using PWV ${ }^{4,5)}$. However, theoretically, PWV is known to depend directly on blood pressure at the time of measurement ${ }^{6}$. Therefore, the validity of PWV is limited especially in studies on hypertension.

Hasegawa $^{7)}$ intended to overcome this limitation of PWV and obtained a nomogram to adjust the PWV value at $80 \mathrm{mmHg}$ of diastolic pressure ${ }^{7)}$. This adjusted PWV was called Hasegawa's PWV (H-PWV), and it represented PWV from the origin of the aorta to the inguinal artery. H-PWV has been used since 1987, and sufficient data have been accumulated in

Address for correspondence: Tomoyuki Yamamoto, Department of Research and Development 1st, Fukuda Denshi Co., Ltd. 2-35-8 Hongo, Bunkyou-ku, Tokyo 113-8420, Japan E-mail: tomoyuki.yamamoto@fukuda.co.jp

Received: May 17, 2018 Accepted for publication: September 11, 2018

Copyright@2019 Japan Atherosclerosis Society

This article is distributed under the terms of the latest version of CC BY-NC-SA defined by the Creative Commons Attribution License. 
Japan $^{7)}$. H-PWV values increase with aging ${ }^{7)}$. However, H-PWV was an artificially adjusted value to avoid the influence of the blood pressure at measuring time. A new index reflecting intrinsic arterial stiffness had therefore been sought for a long time.

The Cardio-Ankle Vascular Index (CAVI), which reflects proper stiffness of the arterial tree from the origin of the aorta to the ankle, was developed in $2004^{8)}$. The unique feature of the index is its independence from blood pressure at the time of measurement. CAVI was derived from the Stiffness Parameter $\beta$ theory proposed by Hayashi ${ }^{9)}$, and this was combined with the Bramwell-Hill formula ${ }^{10)}$. Stiffness Parameter $\beta$ is independent of blood pressure, and CAVI took over this principle. Independency from blood pressure was demonstrated by experimental pharmacological studies using metoprolol, a $\beta 1$ selective blocker ${ }^{11}$.

The CAVI equation includes the coefficients "a" and " $b$ " to adjust it to the value of H-PWV, which is compensated for $80 \mathrm{mmHg}$ of diastolic pressure. The reason for the inclusion of " $a$ " and " $b$ " in the CAVI equation is because at the time when CAVI was developed, H-PWV was considered the gold standard of arterial stiffness with significant clinical data in Japan, and it was well correlated with age. Therefore, we proposed to adjust CAVI values to $\mathrm{H}$-PWV values depending on the patient's age.

Many studies featuring CAVI have been published by European, American, Chinese, Korean, Thailand, and Japanese researchers. Presently, about 20,000 units of VaSera, the equipment used to measure CAVI, are in clinical use worldwide.

CAVI is recognized as a surrogate arteriosclerosis maker that is widely used in clinical practice ${ }^{12)}$ because it shows a high value with aging ${ }^{13,14)}$ and various arteriosclerotic diseases such as coronary artery diseases ${ }^{15,16)}$, cerebral infarction ${ }^{17)}$, and chronic kidney disease ${ }^{18,19)}$. Furthermore, most patients with coronary risk factors have an elevated CAVI ${ }^{12)}$.

A recent study by Yamamoto et al. ${ }^{20)}$ on the role of measuring segmental arterial stiffness using the CAVI theory showed that the application of the CAVI theory to various arterial segments was possible, and that the comparison of those values provides several useful insights. It is expected that applying CAVI theory to various arterial segments will develop new fields in the measurement of arterial stiffness. For that purpose, CAVI without the coefficients "a" and "b" will be necessary because those coefficients were designed for the section from heart to ankle, and the property of each arterial segment is different.

\section{Aim}

The values of the coefficients " $a$ " and " $b$ " have not been disclosed before. To stimulate research in the field of arterial stiffness, and in the spirit of sharing scientific information, we intend to disclose key elements of the CAVI formula, including the coefficients "a" and "b" in this article.

One major concern following this disclosure is the change in the interpretation of CAVI in various studies. To investigate this possibility, a comparison of CAVI with and without the coefficients "a" and " $b$ " (which will hereafter be named heart-to-ankle Beta= ha $\beta$ ) in an epidemiological and a clinical study was performed. The epidemiological study $(n=32,627)$ assesses the significance of CAVI and ha $\beta$ among people with or without coronary risk factors. In this study, the statistical significance of CAVI and ha $\beta$ was assessed in people with coronary risk factors, such as hypertension, dyslipidemia, hyperglycemia, and abnormal electrocardiography (ECG), by comparing values with those of healthy people.

Simple regression analysis and multiple regression analysis were performed with related parameters to compare CAVI and ha $\beta$.

Furthermore, this clinical study compares the effect of nitroglycerin on CAVI and ha $\beta^{21)}$ and the statistical significance of any changes in stiffness.

\section{Methods}

\section{Establishment of the Equation of ha $\beta$ and CAVI} From Stiffness Parameter $\beta$

CAVI is derived from Stiffness Parameter $\beta$, and Bramwell-Hill formula was used to apply this theory to a certain length of the artery.

Bramwell-Hill's formula indicates the relationship between volume elastic modulus and PWV as follows:

$$
\mathrm{PWV}^{2}=\frac{\Delta \mathrm{P}}{\rho} \cdot \frac{\mathrm{V}}{\Delta \mathrm{V}}
$$

Where $\Delta \mathrm{P}$ is pulse pressure, $\mathrm{V}$ is volume of the blood vessel, $\Delta \mathrm{V}$ is the change of $\mathrm{V}$, and $\rho$ is the blood density.

From eq.1, the following equation is derived, when $\Delta$ $\mathrm{D} / \mathrm{D}$ is small:

$$
\mathrm{PWV}^{2}=\frac{\Delta \mathrm{P}}{\rho} \cdot \frac{\mathrm{D}}{2 \Delta \mathrm{D}}
$$

Where $\mathrm{D}$ is the diameter of the blood vessel and $\Delta \mathrm{D}$ is the change of $\mathrm{D}$.

Next, the stiffness parameter $\beta$ represents the following equation: 


$$
\beta=\ln \left(\frac{\mathrm{Ps}}{\mathrm{Pd}}\right) \cdot \frac{\mathrm{D}}{\Delta \mathrm{D}}
$$

Where Ps is systolic blood pressure and Pd is diastolic blood pressure.

When $\mathrm{D} / \Delta \mathrm{D}$ of the eq. 2 is substituted for that in eq.3, the following equation arises:

$$
\beta=\ln \left(\frac{\mathrm{Ps}}{\mathrm{Pd}}\right) \times \frac{2 \rho}{\Delta \mathrm{P}} \times \mathrm{PWV}^{2}
$$

Thus, Stiffness Parameter $\beta$ can be combined with PWV and $\rho$. When PWV is measured between the origin of the aorta to the ankle, the new stiffness index is presented as ha $\beta$.

$$
\text { ha } \beta=\ln \left(\frac{\mathrm{Ps}}{\mathrm{Pd}}\right) \times \frac{2 \rho}{\Delta \mathrm{P}} \times \text { haPWV }^{2}
$$

Where haPWV is heart-ankle PWV.

Finally, CAVI is defined by applying the coefficients "a" and "b" to eq. 5 , as follows ${ }^{8)}$ :

$$
\mathrm{CAVI}=\mathrm{a} \times \ln \left(\frac{\mathrm{Ps}}{\mathrm{Pd}}\right) \times \frac{2 \rho}{\Delta \mathrm{P}} \times \mathrm{haPWV}^{2}+\mathrm{b} \quad \text { eq. } 6
$$

\section{Elements in the CAVI Equation}

a. The Blood Pressure Value Used in the CAVI Equation

There are blood pressure variations between the heart and the ankle. CAVI uses the right upper arm blood pressure as a representative pressure for the whole pathway. When it is difficult to measure blood pressure at the right upper arm, for instance in patients receiving hemodialysis, the left upper arm blood pressure is used.

\section{b. The haPWV Used in the CAVI Equation}

CAVI applies haPWV from the origin of aorta to the ankle. The pulse propagation time from the heart to the ankle is measured by adding tb, the time difference between the second heart sound (II) and the dicrotic notch detected in the pulse wave at the upper right arm cuff, and tba, the time difference between the arrival of the pulse wave at the upper arm cuff and that at the ankle cuff. Thus, by dividing the length of artery by the propagation time, haPWV is calculated by following equation:

$$
\text { haPWV }=\frac{\mathrm{L}}{\mathrm{tb}+\mathrm{tba}}
$$

Where $\mathrm{L}$ is the length of artery, tb is the duration from heart sound II to the dicrotic notch at the upper arm cuff, and tba is the time between the pulse wave arrival point at the upper arm cuff and at the ankle cuff.

Similar to blood pressure measurement, tb, and tba are preferentially measured at the right side. Namely, tb is measured from heart sound II to the dicrotic notch at the cuff of the right upper arm, and " $\mathrm{R}$-tba" is measured with the cuff of the right upper arm and right ankle, "L-tba" is measured at the right upper arm and left ankle. Then, "R-CAVI" and "L-CAVI" are calculated with tb + "R-tba" and tb + "L-tba," respectively.

When only the left upper arm is available because of physical difficulties on the right side, tb is measured with the left upper arm, and "R-CAVI" and "L-CAVI" are calculated in the same manner.

\section{c. The Length of Arterial Tree From the Origin of the Aorta to the Ankle}

The length of the artery is defined as follows: L1: from the aortic valve to the femoral artery, L2: from the femoral to the popliteal artery, and L3: from the popliteal artery to the ankle. $\mathrm{L} 1$ is calculated as $\mathrm{L} 1=$ $1.3 \times \mathrm{AF}$ (Aorta to Femoral) using Nye's method ${ }^{22)}$, where $\mathrm{AF}$ is the direct distance from the sternum at the second intercostal space to the femoral artery at the groin. Then, the total length of the artery from the heart to the ankle is calculated as $\mathrm{L}=\mathrm{L} 1+\mathrm{L} 2+\mathrm{L} 3$.

When using the VaSera device's automatic measurement mode, $\mathrm{L}$ is calculated from the subject's body height using the following equation:

$$
\mathrm{L}=7.7685 \times \text { body height }-17.536
$$

This formula was obtained from the correlation equation between $\mathrm{L}$ and the body height of the 813 subjects at Mihama Hospital.

\section{d. Blood Density $\rho$}

Because the blood density in individuals is generally between 1.045 and $1.055^{23)}$, the fixed value of 1.05 is used in the CAVI equation.

\section{Hasegawa's PWV}

To overcome the limitation of PWV's dependency on blood pressure, Hasegawa et al. created a nomogram of diastolic pressure and PWV by carefully observing their relationship and developed H-PWV using a PWV value corresponding to $80 \mathrm{mmHg}$ of diastolic pressure ${ }^{7)}$. Previous PWV methods had the additional problem of ambiguity in the segment of measurement. H-PWV enabled an accurate measurement by specifying the segment from the origin of the aorta to the femoral artery using a phonocardiogram ${ }^{7}$.

This method was implemented in the PWV-100 (Fukuda Denshi) equipment, which was launched in 1987 as the world's first dedicated apparatus for the measurement of arterial stiffness.

H-PWV was measured in 169 subjects and 


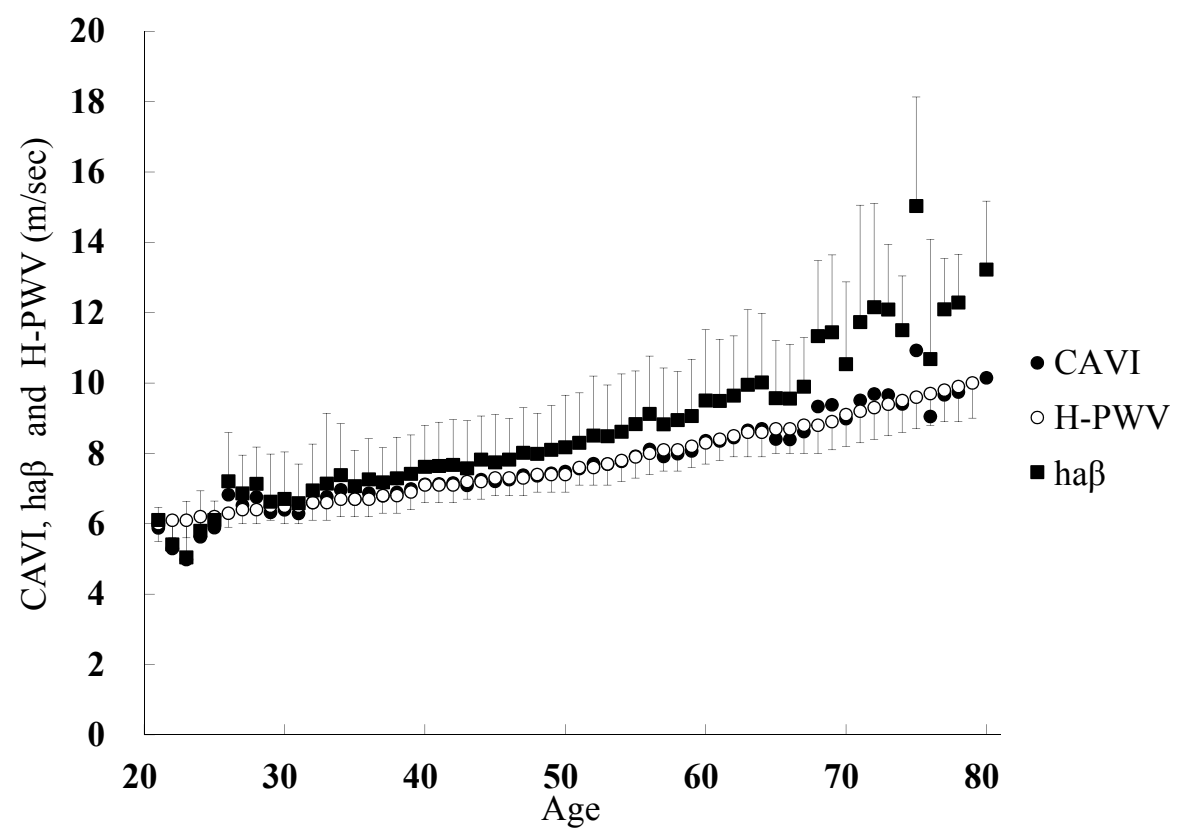

Fig. 1. Average of H-PWV at the Japan Health Promotion Foundation, and ha $\beta$ and CAVI at Osaka Hospital Anti-Tuberculosis Association versus Age

Abbreviations: ha $\beta$, heart-ankle BETA; H-PWV, Hasegawa's pulse wave velocity; CAVI, Cardio-Ankle Vascular Index

results were analyzed to investigate the relationship with cerebral hemorrhage, cerebral infarction, and coronary artery disease. The following results were observed: coronary artery disease: $0 \%$ with no more than $7 \mathrm{~m} / \mathrm{s}$ of PWV, $3.7 \%$ with $8 \mathrm{~m} / \mathrm{s}$, and $24.2 \%$ with $9 \mathrm{~m} / \mathrm{s}$ and over; cerebral artery diseases: $0 \%$ with no more than $7 \mathrm{~m} / \mathrm{s}, 7.7 \%$ with $8 \mathrm{~m} / \mathrm{s}$, and $39.3 \%$ with $9 \mathrm{~m} / \mathrm{s}$ and over; either coronary or cerebral artery disease: $0 \%$ with no more than $7 \mathrm{~m} / \mathrm{s}, 15.4 \%$ with 8 $\mathrm{m} / \mathrm{s}$, and $53.6 \%$ with $9 \mathrm{~m} / \mathrm{s}$ and over. Thus, H-PWV showed a high sensitivity and a capability to appropriately diagnose arteriosclerotic diseases.

Until CAVI was introduced, H-PWV was considered to be the gold standard in Japan, because of its independency of blood pressure ${ }^{7)}$, and because significant data had been accumulated using this method. Therefore, to establish the compatibility between H-PWV and CAVI, we adjusted the CAVI values to H-PWV values with a linear transformation.

For the adjustment, coefficient values "a" and "b" in the CAVI equation were obtained to coincide with the age-dependency of H-PWV, because the age-relationship of H-PWV had already been established.

\section{Adjustment of ha $\beta$ to H-PWV Using Coefficients "a" and "b" in the CAVI Equation}

Arterial stiffness is known to increase with aging. Therefore, "a" and "b" can be determined to corre- spond to age-based change. The averaged ha $\beta$ at each age of the 7,540 subjects who underwent a physical check at Osaka Hospital Anti-Tuberculosis Association was obtained and is shown in Fig. 1 using solid squares.

As for H-PWV, the average values of 106,559 subjects who underwent a physical check at the Japan Health Promotion Foundation are as shown in Fig. 1, using an open circle $\mathrm{e}^{7)}$. Both values increased with age, but the average ha $\beta$ increased much more than H-PWV in the elderly group (more than 60 years).

We decided to perform a linear transformation to adjust ha $\beta$ at the Osaka Hospital Anti-Tuberculosis Association to H-PWV at the Japan Health Promotion Foundation.

Because ha $\beta$ is based on PWV squared, whereas H-PWV is based solely on PWV, it is difficult to maintain a high correlation in a wide range of values with one approximate adjustment. For this reason, the portion that coincides with the correlation equation of ha $\beta$ and H-PWV was defined as the middle range. For the upper and lower ranges that were outside the middle range, the coefficients were adjusted so that the difference between H-PWV and CAVI was small throughout the clinical range.

As a result, we divided the data into three sections (low: ha $\beta<7.34875$, middle, and high: $10.30372 \leq \mathrm{ha} \beta)$, so that the ha $\beta$ could be adjusted 
Table 1. Coefficients "a" and "b" in CAVI

\begin{tabular}{lccc}
\hline ha $\beta$ before transformation & $\begin{array}{c}\text { Low Range } \\
<7.34875\end{array}$ & Middle Range & $\begin{array}{c}\text { High Range } \\
\geq 10.30372\end{array}$ \\
\hline Coefficient a & 0.85 & 0.658 & 0.432 \\
Coefficient b & 0.695 & 2.103 & 4.441 \\
\hline
\end{tabular}

Abbreviations: ha $\beta$, heart-ankle BETA

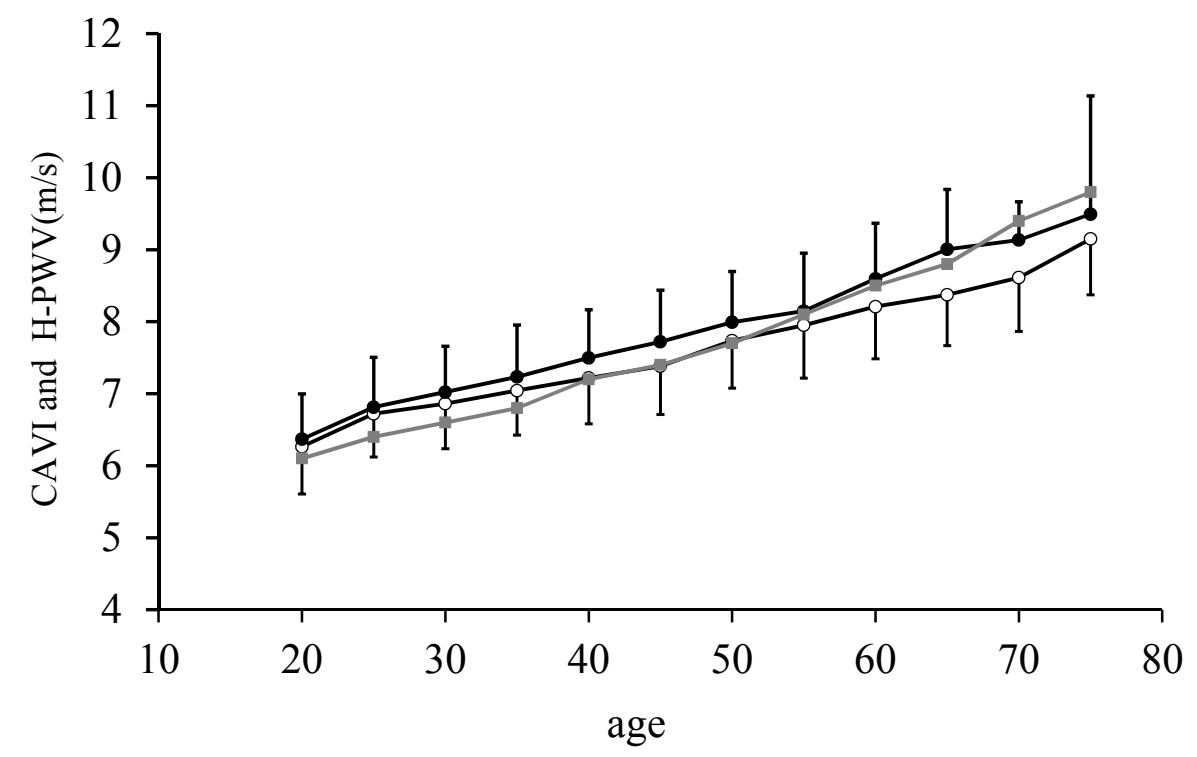

$\rightarrow$ Women $\rightarrow$ Men $\rightarrow$ H-PWV

Fig. 2. Comparison of Average Data Between CAVI of Healthy Subjects, 2,239 Men and 3,730 Women, Total 5,969 among 32,627 who Underwent a Physical Check and the Previous H-PWV at the Japan Health Promotion Foundation Versus Age (5 years each)

for the full range.

The coefficients "a" and "b" are shown in Table 1. Coefficient "a" values were $0.850,0.658$, and 0.432 , respectively, at each of the three ha $\beta$ ranges (low, middle, and high). Coefficient " $b$ " values were 0.695 , 2.103 , and 4.441, respectively, at each of the three ha $\beta$ ranges.

CAVI values including " $a$ " and " $b$ " of the subjects at the Osaka Hospital Anti-Tuberculosis Association are shown in Fig. 1, using a solid circle. As a result, CAVI values corresponded to H-PWV values in each age group.

Although the subjects are not the same, comparison of average data is shown in Fig. 2 (5 years each) between the CAVI values of healthy people, 2,239 men, and 3,730 women (total 5,969) among the 32,627 people who underwent a physical check at the Japan Health Promotion Foundation, and the previous H-PWV at the Japan Health Promotion Foundation, and no large discrepancy was observed.
5. Comparison of the Significant Differences of CAVI and ha $\beta$ Between People With and Without Coronary Risk Factors in the Epidemiological Study

In an epidemiological study, significant differences in the CAVI and ha $\beta$ values between people with and without coronary risk factors such as hypertension, dyslipidemia, hyperglycemia, and ischemic ECG change were investigated. Subjects were selected from the 32,627 patients who underwent a physical check at the Japan Health Promotion Foundation. The flow diagram of this study population is shown in Fig. 3. The criteria are mentioned below ${ }^{24)}$. Healthy group: 2,239 men and 3,730 women with the following criteria: $\mathrm{Ps} \leq 139 \mathrm{mmHg}$ and $\mathrm{Pd} \leq 89 \mathrm{mmHg}$; lipid: $\mathrm{TC} \leq 219 \mathrm{mg} / \mathrm{dL}$ and $40 \leq \mathrm{HDL}-\mathrm{C} \leq 99 \mathrm{mg} / \mathrm{dL}$ and TG $\leq 149 \mathrm{mg} / \mathrm{dL}$; blood Sugar: glucose $\leq 109 \mathrm{mg}$ or HbAlc $\leq 5.8 \%$; renal: creatinine (men) $\leq 1.10 \mathrm{mg} /$ $\mathrm{dL}$, creatinine (women) $\leq 0.80 \mathrm{mg} / \mathrm{dL}$, uric Acid $\leq 7.0$ $\mathrm{mg} / \mathrm{dL}$; white blood cell: $3.2-8.5 \times 103 / \mu \mathrm{L}$; fundus: $\mathrm{S}$ findings - 0 and $\mathrm{H}$ findings - 0 , and ECG: exclude 


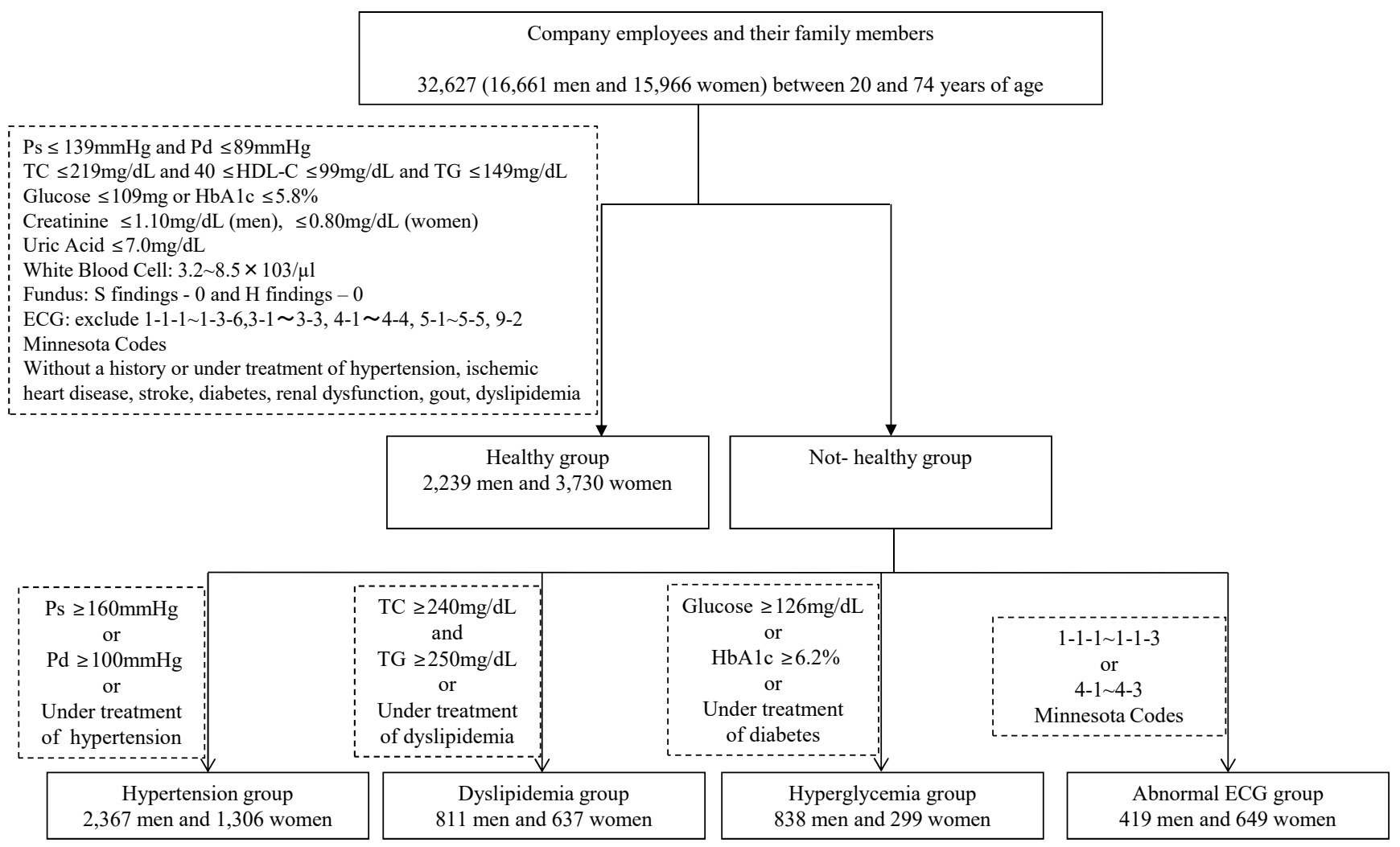

Fig. 3. The Flow Diagram of the Study Population

1-1-1 1-3-6, 3-1 -3-3, 4-1 -4-4, 5-1 - 5-5, 9-2 Minnesota Codes. Furthermore, those with a history or treatment of hypertension, ischemic heart disease, stroke, diabetes, renal dysfunction, gout, and dyslipidemia were excluded.

Hypertension group: 2,367 men and 1,306 women with $\mathrm{Ps} \geq 160 \mathrm{mmHg}$ or $\mathrm{Pd} \geq 100 \mathrm{mmHg}$, or under treatment of hypertension.

Dyslipidemia group: 811 men and 637 women with $\mathrm{TC} \geq 240 \mathrm{mg} / \mathrm{dL}$ and $\mathrm{TG} \geq 250 \mathrm{mg} / \mathrm{dL}$, or under treatment of dyslipidemia.

Hyperglycemia group: 838 men and 299 women with Fasting Glucose $\geq 126 \mathrm{mg} / \mathrm{dL}$ or HbAlc $\geq 6.2 \%$, or under treatment of diabetes.

Abnormal ECG: 419 men and 649 women with any of 1-1-1 1-1-3, 4-1 4-3 Minnesota Codes.

Healthy people and people with the above risk factors were divided into 12 groups by sex and age strata. The age groups were 20-29, 30-39, 40-49, 50-59, 60-69, 70 years, and over. The statistical significance of CAVI values and ha $\beta$ values between healthy people and people with risk factors were calculated, respectively.

Simple regression analysis and multiple regression analysis were performed for CAVI and ha $\beta$ in all healthy people and those with risk factors, and the following parameters' results were compared; Sex, Age, BMI, Ps, Pd, HDL-C, TG, Creatinine, and Glucose. Dummy variables were set in Sex (men: 1, women: 2).

6. Comparison of Significant Differences in the Changes of CAVI and ha $\beta$ during Nitroglycerin Administration in Normal Control Subjects and Coronary Artery Disease (CAD) Patients

Arterial stiffness consists of structural stiffness and functional stiffness. To investigate whether there is a difference between CAVI and ha $\beta$ with the transient change of functional stiffness, the difference between CAVI and ha $\beta$ was analyzed following the administration of nitroglycerin.

We reported a significant decrease of CAVI after administration of nitroglycerin in normal control subjects $(n=31)$ and CAD patients $(n=25)^{21)}$. The ha $\beta$ values obtained in this study, and the statistical significance of the changes of ha $\beta$ values were calculated and compared with those of CAVI.

\section{Statistical Analysis}

Unpaired Welch's $t$-test was used to evaluate comparisons of each parameter among the different 

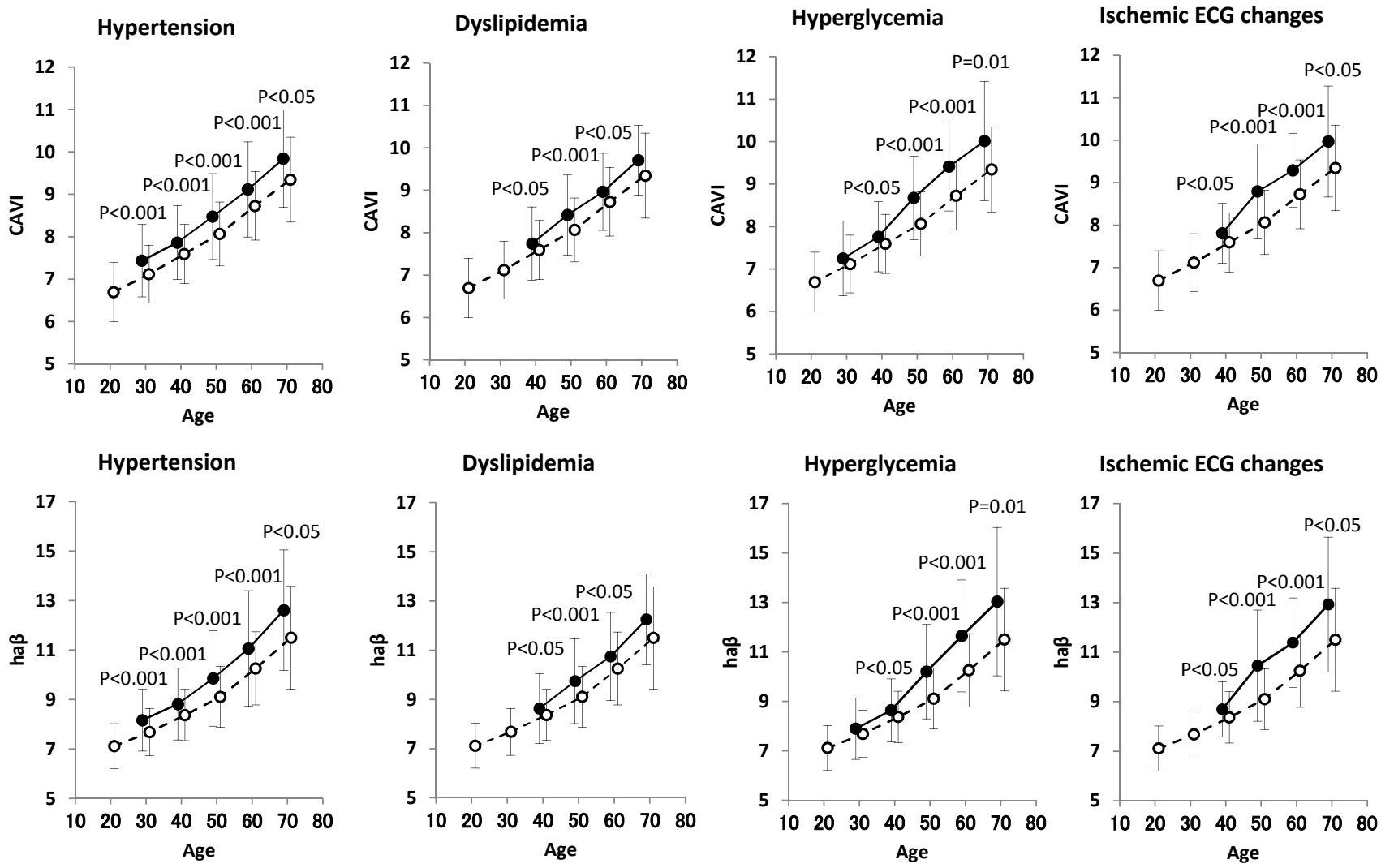

Fig.4. CAVI and ha $\beta$ in the Healthy Group (open circles) and Each Risk Group (solid circles) in men

Notes: Data are presented as mean \pm standard deviation; Unpaired Welch's $t$-test was used to compare healthy groups and each risk group. Abbreviations: CAVI, Cardio-Ankle Vascular Index; ha $\beta$, heart-ankle BETA

coronary risk factor groups in the epidemiological study. Regression analysis was used to compare CAVI and ha $\beta$ in the $\mathrm{R}$ of simple regression and the $\beta$ of multiple regression with each related parameter. Comparisons of each parameter before and after taking nitroglycerin were analyzed using Student $t$-test. We performed post hoc power analysis using the $G^{*}$ Power 3 software (Dusseldorf, Germany) in the nitroglycerin administration study ${ }^{21)}$. The detection power was $77 \%$ $(p<0.05$, two tails) in the normal controls $(n=31)$, and $67 \%$ in the CAD patients $(n=25)$. In both t-tests, results were expressed as the mean \pm standard deviation.

All statistical analyses were double-sided, and $p<$ 0.05 was considered significant. Statistical analysis was performed using the SPSS software package (SPSS Inc., Chicago, IL, USA).

\section{Results}

Comparison of the Significant Differences of CAVI and ha $\beta$ between People with or without Coronary Risk Factors in the Epidemiological Study

The statistically significant differences of CAVI and ha $\beta$ between people with or without coronary risk factors in the epidemiological study are shown in Fig. 4 (men) and Fig. 5 (women).

As for hypertension, CAVI and ha $\beta$ were significantly higher in 30-39 year-old men, but not women. And, both indices were significantly higher in 40-79 year-old men and women. The age groups with a significant difference $(p<0.05)$ were identical between CAVI and ha $\beta$.

Concerning dyslipidemia, CAVI and ha $\beta$ were not significantly higher in 30-39 year-old women. Both indices were significantly higher in 40-69 year-old men and women as well as in 70-79 year-old women, however they were not significantly higher in 70-79 year-old men. The age groups with a significant difference $(p<0.05)$ were identical between CAVI and ha $\beta$.

For hyperglycemia, CAVI and ha $\beta$ were significantly higher in 40-49 year-old men, but not women. And, both indices were significantly higher in 50-79 year-old men and women. The age groups with a significant difference $(p<0.05)$ were identical between CAVI and ha $\beta$.

As for ischemic ECG abnormality, CAVI and 


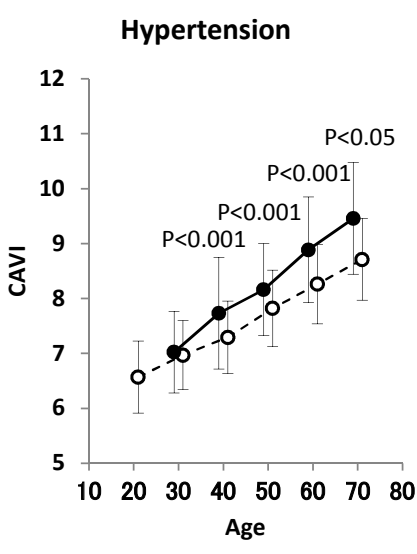

Hypertension

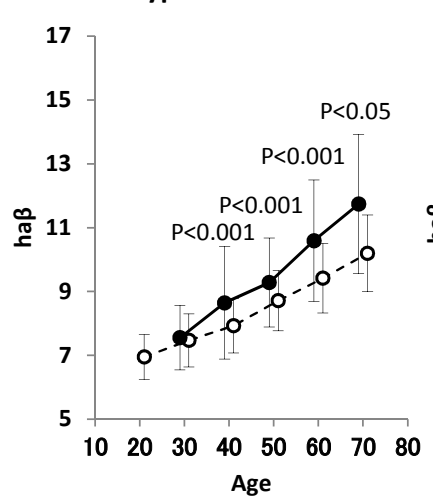

Dyslipidemia

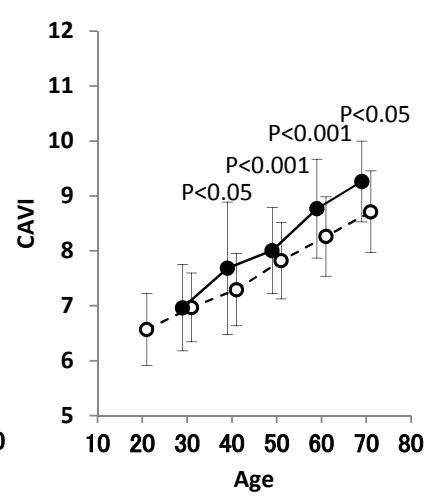

Dyslipidemia

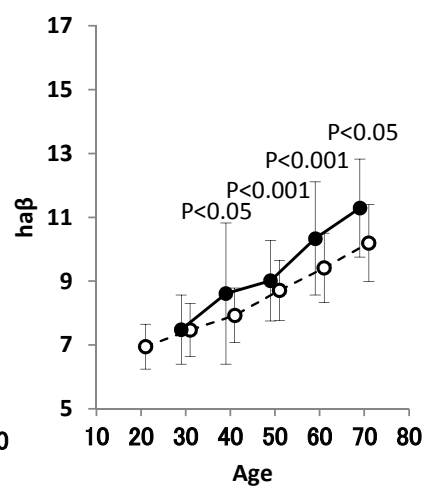

Hyperglycemia

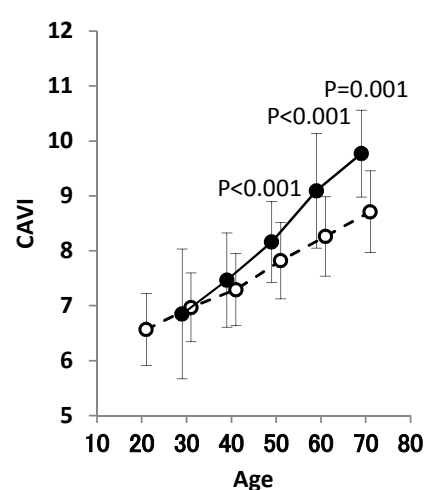

Hyperglycemia

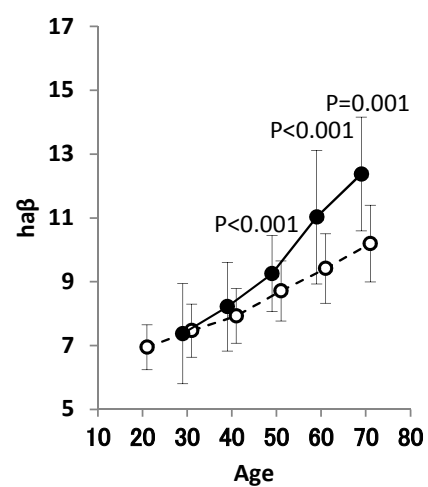

Ischemic ECG changes

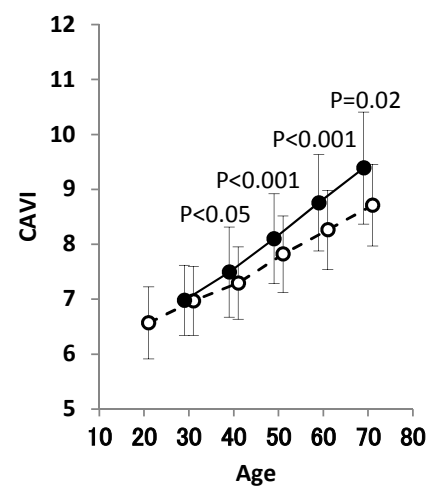

Ischemic ECG changes

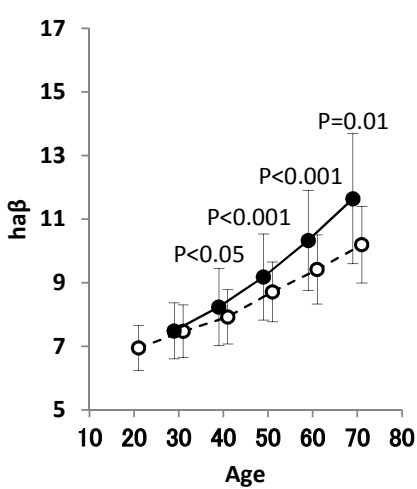

Fig. 5. CAVI and ha $\beta$ in the Healthy Group (open circles) and Each Risk Group (solid circles) in Women

Notes: Data are presented as mean \pm standard deviation; Unpaired Welch's $t$-test was used to compare healthy groups and each risk group. Abbreviations: CAVI, Cardio-Ankle Vascular Index; ha $\beta$, heart-ankle BETA

ha $\beta$ were not significantly higher in the 30-39 yearold women. And, both indices were significantly higher in 40-79 year-old men and women. Again, the age groups with a significant difference $(p<0.05)$ were identical between CAVI and ha $\beta$.

In summary, the statistical significance of higher CAVI and ha $\beta$ values in people with hypertension, dyslipidemia, hyperglycemia and abnormal ECG compared with those in the healthy people matched at each age in men and women.

Regression analysis was performed, and no substantial discrepancy was found between CAVI and ha $\beta$ in the $\mathrm{R}$ of simple regression and the $\beta$ of multiple regression analysis (Table 2).

Comparison of the Significant Differences of the Changes of CAVI and ha $\beta$ during Nitroglycerin Administration in Normal Control Subjects and CAD Patients

Nitroglycerin administration decreased CAVI values over a period of $20 \mathrm{~min}$. as reported by Shimizu et al. ${ }^{21)}$. The CAVI and ha $\beta$ values were obtained from this study and those values at 5, 10, 15, and $20 \mathrm{~min}$. were compared with baseline values and these are shown in Fig. 6 (normal controls) and Fig. 7 (CAD patients).

The $p$ values in each group were less than 0.05 , and no discrepancy in terms of statistically significant differences was found between CAVI and ha $\beta$ in the change of values from baseline.

\section{Discussion}

CAVI was developed to overcome the problem of the blood pressure dependency of $\mathrm{PWV}^{8)}$. The idea was to create an index reflecting the proper arterial stiffness of the arterial tree from the origin of the aorta to the ankle. When CAVI was developed, plenty of data on H-PWV had already been collected and analyzed. To compare the values of CAVI with the existing data of H-PWV, CAVI values were adjusted to H-PWV at different ages by including coefficients "a" 
Table 2. Simple Regression and Multiple Regression of CAVI and ha $\beta$ with Related Parameters

\begin{tabular}{|c|c|c|c|c|c|c|c|c|}
\hline & \multicolumn{4}{|c|}{ CAVI } & \multicolumn{4}{|c|}{ ha $\beta$} \\
\hline & \multicolumn{2}{|c|}{ Simple Regression } & \multicolumn{2}{|c|}{ Multiple Regression } & \multicolumn{2}{|c|}{ Simple Regression } & \multicolumn{2}{|c|}{ Multiple Regression } \\
\hline & $r$ & $p$ value & $\beta$ & $p$ value & $r$ & $p$ value & $\beta$ & $p$ value \\
\hline Age & 0.697 & $<0.01$ & 0.648 & $<0.001$ & 0.676 & $<0.01$ & 0.620 & $<0.001$ \\
\hline Sex & -0.142 & $<0.01$ & -0.094 & $<0.001$ & -0.139 & $<0.01$ & -0.085 & $<0.001$ \\
\hline BMI & -0.021 & $<0.01$ & -0.208 & $<0.001$ & -0.013 & 0.12 & -0.198 & $<0.001$ \\
\hline Ps & 0.354 & $<0.01$ & 0.128 & $<0.001$ & 0.360 & $<0.01$ & 0.143 & $<0.001$ \\
\hline HDL-C & -0.020 & 0.01 & -0.026 & $<0.001$ & -0.024 & $<0.01$ & -0.030 & $<0.001$ \\
\hline TG & 0.098 & $<0.01$ & 0.031 & $<0.001$ & 0.094 & $<0.01$ & 0.023 & 0.001 \\
\hline Glucose & 0.248 & $<0.01$ & 0.063 & $<0.001$ & 0.250 & $<0.01$ & 0.070 & $<0.001$ \\
\hline
\end{tabular}

Abbreviations: ha $\beta$, heart-ankle BETA
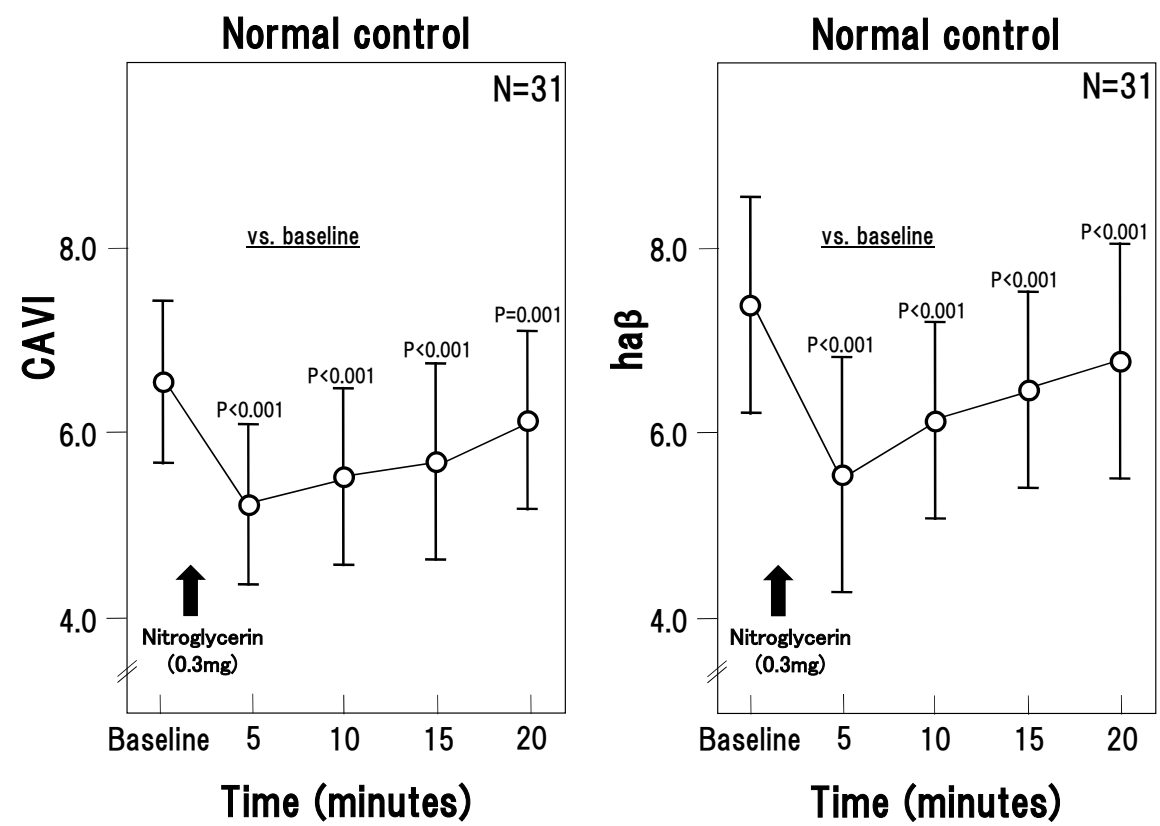

Fig. 6. Changes in CAVI after Sublingual Administration of Nitroglycerin $0.3 \mathrm{mg}$ in Normal Controls $(n=31)$.

Notes: CAVI was measured using VaSera at baseline, 5, 10, 15, and 20 min after the administration of nitroglycerin $0.3 \mathrm{mg}$, and the value on the right side of CAVI was used. Data are presented as mean \pm standard deviation; Student's $t$-test was used to compare baseline and after administration.

Abbreviations: CAVI, Cardio-Ankle Vascular Index; ha $\beta$, heart-ankle BETA

and " $b$ " in the CAVI equation. As a result, CAVI succeeded in largely matching H-PWV in age-dependency. Originally, arterial stiffness measurements were not limited to indices of vascular aging alone, but also used as indices to measure the functional stiffness of the arterial wall. We have disclosed the coefficients "a" and " $b$ " to stimulate research in the field of arterial stiffness.

One concern, that the significance or interpretations of CAVI with and without coefficients "a" and "b" (ha $\beta$ ) might cause differences in epidemiological studies and clinical studies, was raised.

To assuage this concern two studies were performed.

In the epidemiological study $(n=32,627)$, the statistical significance between CAVI and ha $\beta$ were essentially the same in people with higher coronary risks such as hypertension, dyslipidemia, hyperglycemia, and abnormal ECG compared with those of healthy men and women in each age group. Also, no 

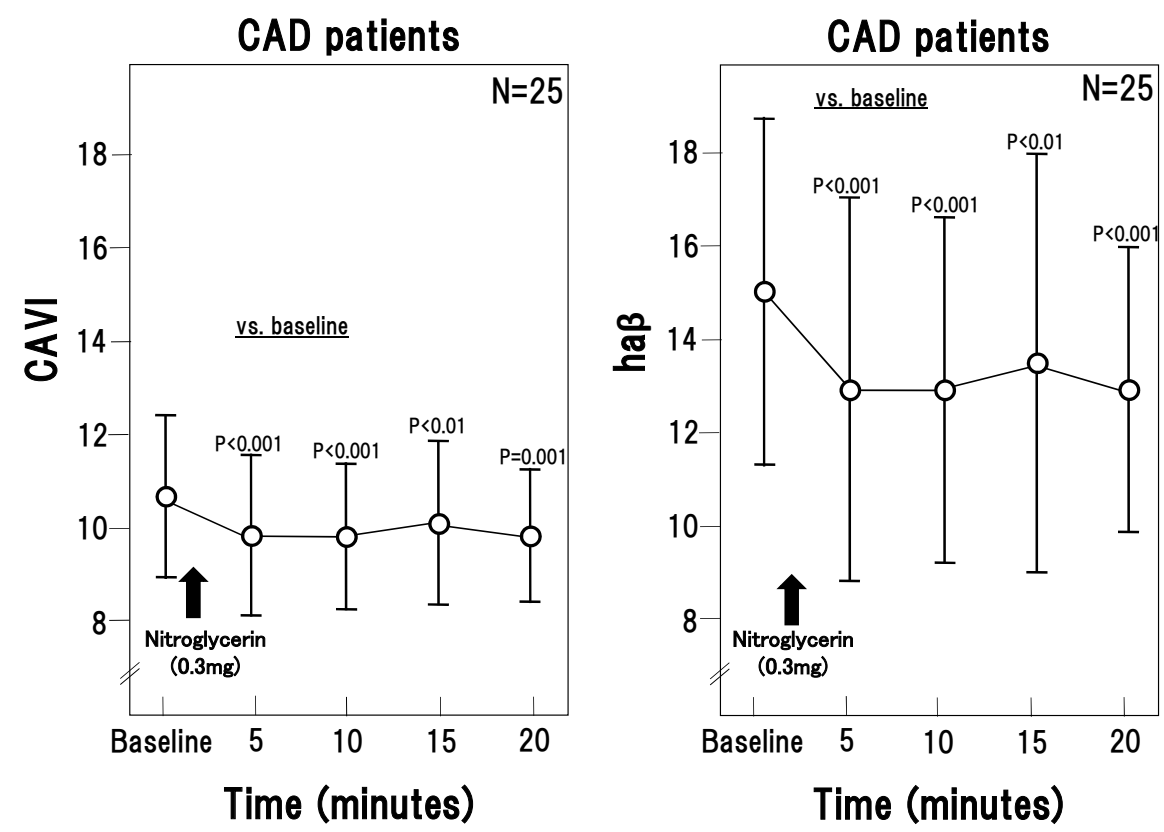

Fig. 7. Changes in CAVI after Sublingual Administration of Nitroglycerin $0.3 \mathrm{mg}$ in CAD Patients $(n=25)$.

Notes: CAVI was measured using VaSera at baseline, 5, 10, 15, and $20 \mathrm{~min}$ after the administration of nitroglycerin $0.3 \mathrm{mg}$, and the value on the right side of CAVI was used. Data are presented as mean \pm standard deviation; Student's $t$-test was used to compare baseline and after administration.

Abbreviations: CAVI, Cardio-Ankle Vascular Index; ha $\beta$, heart-ankle BETA

substantial discrepancy was found in the simple regression and multiple regression analysis with related parameters. These results suggest that the CAVI and ha $\beta$ are essentially the same in people with those risk factors.

In the clinical study, changes in CAVI and ha $\beta$ values were compared after the administration of nitroglycerin in normal controls and CAD patients. There was no discrepancy between the statistical significances of the changes in CAVI and ha $\beta$ in normal controls and in CAD patients.

In conclusion, there is no concern that the significance or interpretation of CAVI and ha $\beta$ would be different in either epidemiological studies or clinical studies.

With this publication, all of the previously undisclosed information of CAVI has been revealed including coefficients "a" and "b", and the calculation method of the arterial length based upon a subject's height.

Studies using ha $\beta$ are also now possible.

We sincerely hope this publication contributes to the progress of clinical research in the field of both organic and functional arterial stiffness.

\section{Disclosures}

Koji Takahashi, Tomoyuki Yamamoto, and Shinichi Tsuda belong to Fukuda Denshi Co. Ltd and are involved in the development of CAVI.

Fumio Okabe, Tadashi Shimose, Yoshinori Tsuji, Kenji Suzuki, Kuniaki Otsuka, Masanobu Takata, Kazuhiro Shimizu, Junji Uchino, and Kohji Shirai have no conflict of interest concerning this paper.

\section{Acknowledgments}

We thank the late Motoharu Hasegawa for providing valuable suggestions during the development of CAVI.

\section{References}

1) Laurent S, Boutouyrie P, Asmar R, Gautier I, Laloux B, Guize L, Ducimetiere P, Benetos A. Aortic stiffness is an independent predictor of all-cause and cardiovascular mortality in hypertensive patients. Hypertension, 2001; 37: 1236-1241

2) Laurent S, Katsahian S, Fassot C, Tropeano AI, Gautier I, Laloux B, Boutouyrie P. Aortic stiffness is an independent predictor of fatal stroke in essential hypertension. Stroke, 2003; 34: 1203-1206 
3) Benetos A, Waeber B, Izzo J, Mitchell G, Resnick L, Asmar R, Safar M. Influence of age, risk factors, and cardiovascular and renal disease on arterial stiffness: clinical applications. Am J Hypertens, 2002; 15: 1101-1108

4) Laurent S, Boutouyrie P, Asmar R, Gautier I, Laloux B, Guize L, Ducimetiere P, Benetos A. Aortic stiffness is an independent predictor of all-cause and cardiovascular mortality in hypertensive patients. Hypertension, 2001; 37: 1236-1241

5) Vlachopoulos C, Aznaouridis K, Stefanadis C. Prediction of cardiovascular events and all-cause mortality with arterial stiffness: a systematic review and meta-analysis. J Am Coll Cardiol, 2010; 55: 1318-1327

6) Avolio AP, Chen SG, Wang RP, Zhang CL, Li MF, O'Rourke MF. Effects of aging on changing arterial compliance and left ventricular load in a northern Chinese urban community. Circulation, 1983; 68: 50-58

7) Hasegawa M, Chikao Arai. Clinical Estimation of Vascular Elastic Function and Practical Application. Connective Tissue, 1995; 27: 149-157

8) Shirai K, Utino J, Otsuka K, Takata M. A novel blood pressure-independent arterial wall stiffness parameter; cardio-ankle vascular index (CAVI). J Atheroscler Thromb, 2006 Apr; 13(2): 101-107

9) Hayashi K, Handa H, Nagasawa S, Okumura A, Moritake K. Stiffness and elastic behavior of human intracranial and extracranial arteries. J Biomech, 1980; 13(2): 175-184

10) Bramwell JC, Hill AV. Velocity of transmission of the pulse and elasticity of arteries. Lancet, 1922; 199: 891892

11) Shirai K, Song M, Suzuki J, Kurosu T, Oyama T, Nagayama D, Miyashita Y, Yamamura S, Takahashi M. Contradictory effects of $\beta 1$ - and $\alpha 1$ - adrenergic receptor blockers on cardio-ankle vascular stiffness index (CAVI) -the independency of CAVI from blood pressure. J Atheroscler Thromb, 2011; 18: 49-55

12) Kozaburo Hayashi, Tomoyuki Yamamoto, Akira Takahara, Kohji Shirai. Clinical assessment of arterial stiffness with cardio-ankle vascular index: theory and applications. J Hypertens, 2015; 33: 1742-1757

13) Takaki A, Ogawa H, Wakeyama T, Iwami T, Kimura M, Hadano Y, Matsuda S, Miyazaki Y, Matsuda T, Hiratsuka A, Matsuzaki M. Cardio-ankle vascular index is a new noninvasive parameter of arterial stiffness. Circ J, 2007; 71: $1710-1714$
14) Namekata T, Suzuki K, Ishizuka N, Shirai K. Establishing baseline criteria of cardio-ankle vascular index as a new indicator of arteriosclerosis: a cross-sectional study. BMC Cardiovasc Disord, 2011; 11: 51

15) Nakamura K, Tomaru T, Yamamura $S$, Miyashita $Y$, Shirai $\mathrm{K}$, Noike H. Cardio-ankle vascular index is a candidate predictor of coronary atherosclerosis. Circ J, 2008; 72: 598-604

16) Horinaka $S$, Yabe A, Yagi $H$, Ishimura $K$, Hara $H$, Iemura T, Matsuoka H. Comparison of atherosclerotic indicators between cardio ankle vascular index and brachial ankle pulse wave velocity. Angiology, 2009; 60: 468-476

17) Suzuki J, Sakakibara R, Tomaru T, Tateno F, Kishi M, Ogawa E, Kurosu T, Shirai K. Stroke and cardio-ankle vascular stiffness index. J Stroke Cerebrovasc Dis, 2011; 22: $171-175$

18) Nakamura K, Iiduka T, Takahashi M, Shimizu S, Mikamo H, Nakagami T, Suzuki M, Hirano K, Sugiyama Y, Tomaru T, Miyashita Y, Shirai K, Noike H. Association between cardio-ankle vascular index and serum cystatin $\mathrm{c}$ levels in patients with cardiovascular risk factor. J Atheroscler Thromb, 2009; 16: 371-379

19) Kubozono T, Miyata H, Uegama K, Nagaki A, Hamasaki S, Kusano K, Kubozono O, Tei C. Association between arterial stiffness and estimated glomerular filtration rate in the Japanese general population. J Atheroscler Thromb, 2009; 16: 840-845

20) Yamamoto T, Shimizu K, Takahashi M, Tatsuno I, Shirai K. The Effect of Nitroglycerin on Arterial Stiffness of the Aorta and the Femoral-Tibial Arteries. J Atheroscler Thromb, 2017; 24: 1048-1057

21) Shimizu K, Yamamoto T, Takahashi M, Sato $S$, Noike H, Shirai K. Effect of nitroglycerin administration on cardioankle vascular index. Vasc Health Risk Manag, 2016; 12: 313-319

22) Nye E. R. The effect of blood pressure alteration on the pulse wave velocity. Br Heart J, 1964; 26: 261-265

23) Nakamori Eizi. An investigation on hematological measurements for screening purpose. Jap J Ind Health, 1971; 13: 191-212 (in Japanese)

24) Suzuki K, Ishizuka N, Miyashita Y, Shirai K. Epidemiological examination about the standard value and the validity for the standardization as the examination of CAVI (cardio-ankle vascular index) noninvasive blood pressure-independent arteriosclerosis test. Niigata J Med Technol, 2008; 48-1: 2-10; (in Japanese) 